\title{
Impacts of combined osteopenia/osteoporosis and sarcopenia on balance and quality of life in older adults
}

\author{
D Raikan Buyukavci, ${ }^{1}$ (i) Semra Akturk, ${ }^{1}$ (i) Bahri Evren, ${ }^{2}$ (i) Yuksel Ersoy $^{1}$ \\ ${ }^{1}$ Department of Physical Medicine and Rehabilitation, Inonu University Faculty of Medicine, Malatya, Turkey \\ ${ }^{2}$ Department of Endocrinology, Inonu University Faculty of Medicine, Malatya, Turkey
}

\begin{abstract}
OBJECTIVE: Combined osteopenia/osteoporosis and sarcopenia is a major public health problem for old adults. In this study, we aimed to evaluate the impacts of combined osteopenia/osteoporosis with sarcopenia on balance and quality of life in patients older than 65 years.

METHODS: In this sudy, 77 patients with sarcopenia, who were older than 65 years, were included. The diagnosis of sarcopenia was made according to the diagnostic criteria developed by The European Working Group on Sarcopenia in Older People (EWGSOP). Bone densitometry was performed to screen for osteoporosis or osteopenia. The balance was assessed with the anterior-posterior stability index (APSI), medial-lateral stability index (MLSI), and the general stability index (OSI), which were calculated using a Biodex Stability System device (BSS). The quality of life was assessed using SF-36.

RESULTS: Patients with sarcopenia were included in this study. Of them, 40 had osteoporosis and 37 had osteopenia. The measures of balance and the OSI, APSI, and MLSI values were low in both groups of patients, but they were statistically significantly lower in the sarcopenia with osteoporosis group compared to the sarcopenia with osteopenia group ( $p=0.01$; $p=0.002 ; p=0.04$, respectively). The quality of life was lower in all sub-categories of SF-36, excluding the mental health when sarcopenia was accompanied by osteoporosis compared to the joint occurrence of sarcopenia with osteopenia $(p<0.05)$.

CONCLUSION: Our study suggests that the joint occurrence of osteoporosis with sarcopenia is associated with a risk of balance loss, a decrease in quality of life, and a potentially increased fracture risk in older adults.

Keywords: Balance; osteoporosis; quality of life; sarcopenia.

Cite this article as: Buyukavci R, Akturk S, Evren B, Ersoy Y. Impacts of combined osteopenia/osteoporosis and sarcopenia on balance and quality of life in older adults. North Clin Istanb 2020;7(6):585-590.
\end{abstract}

Sarcopenia is a disorder with generalized and progressive loss of muscle mass, strength, and function. It is a disease of old individuals primarily [1]. Alterations in the body composition with loss of muscle mass and function occur in time with increasing age. These changes may lead to poor physical performance, weakness, mobility impairments, loss of balance, falls, and disability, eventually becoming severe health problems in aging individuals. The prevalence of sarcopenia in community-based studies ranges from $1 \%$ to $29 \%$. This rate is approximately $10 \%$ of the old people [2]. In 2017, a study from Turkey investigating the prevalence of sarcopenia by the decades reported that sarcopenia prevalence was $15.4 \%$ in the age group of $60-69$ years and that it increased to $36.5 \%$ after 80 years [3].

Received: October 11, 2019 Accepted: May 04, 2020 Online: October 01, 2020

Correspondence: Raikan BUYUKAVCI, MD. Inonu Universitesi Tip Fakultesi, Fiziksel Tip ve Rehabilitasyon Anabilim Dali, Malatya, Turkey.

Tel: +90 5054920264 e-mail: rsoydemir@yahoo.com

(c) Copyright 2020 by Istanbul Provincial Directorate of Health - Available online at www.northclinist.com 
It has been demonstrated that walking speed declines gradually in old individuals. The decline in walking speed is more pronounced in the presence of sarcopenia [4]. Walking speed is a major diagnostic criterion of sarcopenia, defining walking speed almost as a vital sign. Sarcopenia diagnosis is made in old individuals according to the sarcopenia algorithm recommended by The European Working Group on Sarcopenia in Older People (EWGSOP)[5].

Several factors causing sarcopenia are involved in the development of osteoporosis, too. These factors include reduced physical activity, protein-poor diet, chronic inflammatory processes, and hormonal changes [6]. It is reported that individuals with repeated falls in medical history develop a fear of falling and they start to exhibit avoidance behavior toward the activities of daily living, resulting in an eventual reduction in mobility. Therefore, it can be suggested that a vicious cycle starts with a reduction in physical performance and balance loss in old individuals due to sarcopenia and contributing osteoporosis; resulting in fear of falls, which leads to a gradual decrease in physical activities, completing the vicious cycle with more pronounced sarcopenia and osteoporosis [7].

Muscles and bones are considered to compose a functional unit not only because of their close anatomical neighborhood but due to their involvement with the paracrine and endocrine signaling as well. Anatomical, cellular, and biochemical changes occur in the muscle tissue along with aging and these are associated with overlapping similar pathways involved in the alterations of bone mineral density and bone microarchitecture.

Osteoporosis and sarcopenia are associated with increased morbidity and mortality in the older population. With the increased average life expectancy of the world's population, the concept of quality of life in old individuals has become one of the most important goals. Identifying the relationship between osteoporosis and sarcopenia is of considerable importance in developing preventive approaches for people at advanced ages.

In this study, we aimed to investigate the impacts of combined osteopenia/osteoporosis and sarcopenia on balance and quality of life in old individuals.

\section{MATERIALS AND METHODS}

Study Design and Population

The study sample included individuals; who were at least 65 years old and who were admitted to our outpatient clinic. This study was approved by the local ethics committee $(2017 / 75)$.

The inclusion criteria for this study required the individuals to be 65 years old or older and score at least 17 on the Mini-Mental State Examination [8]. The exclusion criteria were the inability to move without assistance, the self-reported presence of acute pain in the lower or upper limbs, physical disability, cardiac or respiratory disorders, history of stroke, cancer cachexia, medication-induced anorexia, an untreated chronic disease or mental illness, severe arthritis, and/or inflammatory diseases. Informed consent was obtained from all participants included in this study.

\section{Measurements}

\section{Clinical Chemistry}

Serum levels 25-OH-vitamin D3 were analyzed using an on-line SPE HPLC device compatible with tandem mass spectrometry (LC-MS/MS). MS7100 and MS7000 ClinMass ${ }^{\circledR}$ Complete Kits were used.

\section{Balance Test with the Biodex Stability System(BSS)}

Biodex Stability System (BSS) (Biodex Medical Systems, Shirley, 2000, New York) was used for quantifying the balance parameters in this study. Antero-Posterior Stability Index (APSI), Medial-Lateral Stability Index (MLSI) and Overall Stability Index (OSI) were tested by an expert while the study participants were undergoing the test with their eyes open. The foot platform of BSS could be set at eight levels by changing the resistance level. Level 8 provided the most stable and level 1 provided the least stable platforms. The measurements were made on the balance platform with the participants on bare feet opened at the width of their shoulders, their knees $15^{\circ}$ twisted, and their hands combined on the chest. The test started at level 8 and ended at level 4. A high value indicated that a substantial movement occurred far from the patient's center of balance, while a low number indicated that a minimal movement occurred during the test [9]. To reduce the fear of falling, the individuals were convinced that they would not fall during the test on the mobile platform. Until the test was completed, an observer continued to stand next to the device. None of the individuals fell during the test (Fig. 1).

\section{Definition of Osteopenia and Osteoporosis}

Total and regional bone mass density (BMD) $\left(\mathrm{g} / \mathrm{cm}^{2}\right)$ and total and regional lean mass were evaluated by du- 


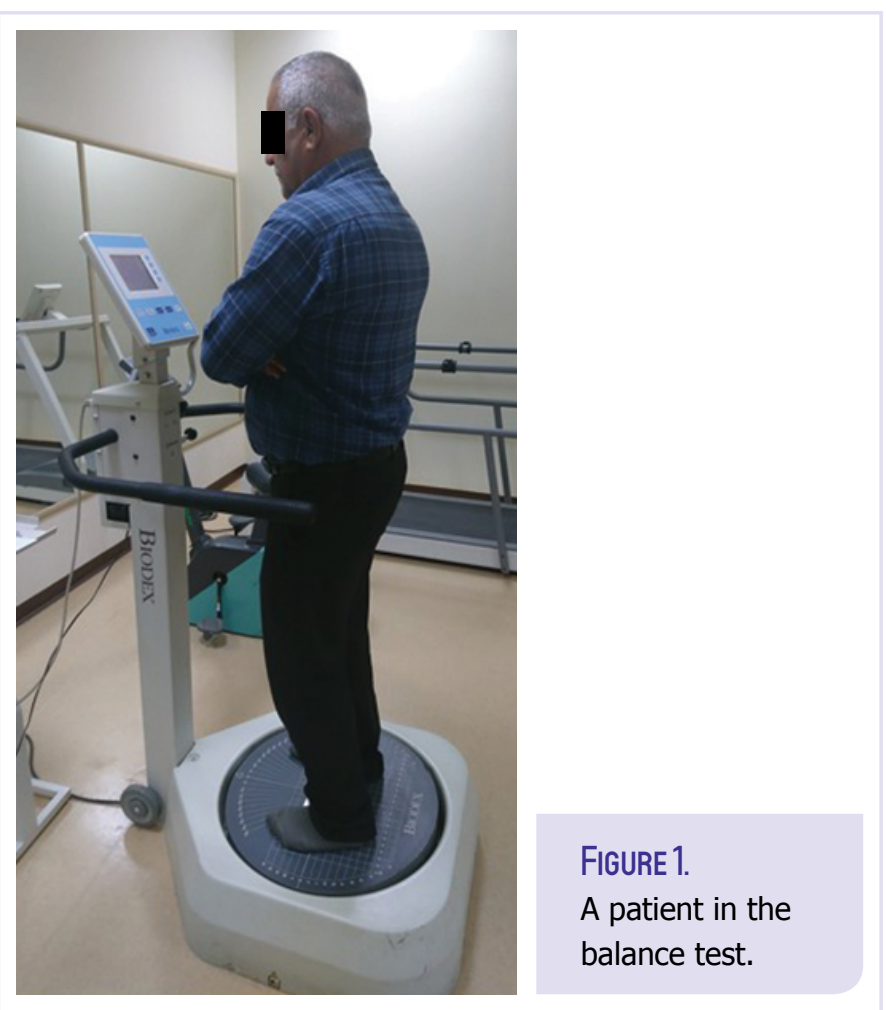

al-energy X-ray absorptiometry (DEXA) [Hologic QDR $4500 \mathrm{~W}$ (SIN 49584), Waltham, MA, USA]. BMD was classified according to the World Health Organization (WHO) criteria based on the T-score of the lumbar spine and/or femoral neck and/or total hip as follows: normal (T-score $>-1.0 \mathrm{SD})$, osteopenia $(-1.0 \geq \mathrm{T}$-score $>-2.5 \mathrm{SD}$ ), and osteoporosis (T-score $\leq-2.5 \mathrm{SD})[10]$.

\section{Definition of Sarcopenia}

We used the definition and algorithm of sarcopenia developed by EWGSOP to identify individuals with sarcopenia [5]. According to this algorithm, firstly, the walking speed was determined in individuals over 65 years. A walking speed of less than $0.8 \mathrm{~m} / \mathrm{sec}$ indicated the risk of sarcopenia, indicating that further evaluation was needed with the handgrip strength test (HGS). If the HGS test results were low, too, then, a selected method of muscle mass measure should be used.

All participants performed a 3-meter-walking speed test and the results were expressed in meters per second. The participants' performance was determined by referencing the cut-off points recommended by the EWGSOP [5]. A walking speed of $\leq 0.8 \mathrm{~m} / \mathrm{s}$ was considered to indicate an impaired physical performance. Muscle strength was assessed by measuring HGS with a Jamar hydraulic hand dynamometer using a validated protocol
[11]. HGS was measured when the participant was in the sitting position with the elbow in $90^{\circ}$ flexion and the wrist in the neutral position. Patients were asked to apply the maximum grip strength three times with both hands. At least a 30 second-period was allowed between each testing episode. The maximum value obtained during the HGS tests of each participant was accepted as the grip strength value to be included in the data analysis [11]. HGS cutoff values were reported to be $<32 \mathrm{~kg}$ and $<22 \mathrm{~kg}$ in males and females, respectively, in the Turkish population [12].

Body composition was evaluated with bioimpedance analysis (BIA) using a Tanita BC 532 model body analysis monitor. BIA predicts the volume of fat and lean body mass. The test itself is not costly, easily administered, and appropriate for ambulatory patients. The results are reported to be reproducible. In our study, we measured the fat-free mass (FFM) with BIA. Then, we calculated the skeletal muscle mass (SMM) using the following equation: SMM $(\mathrm{kg})=0.566 *$ FFM (lean mass). Skeletal muscle mass index (SMMI) was calculated using the following formula: Skeletal muscle mass $(\mathrm{kg}) /$ height squared $\left(\mathrm{m}^{2}\right)$ [13]. The cut-off values for SMMI were $9.2 \mathrm{~kg} / \mathrm{m}^{2}$ in males and $7.4 \mathrm{~kg} / \mathrm{m}^{2}$ in females [12].

\section{Quality of Life}

Short-Form Health Survey (SF-36) was used for assessing the quality of life [14]. SF-36 was previously translated into Turkish and validated for use in the Turkish population. SF-36 is a self-report generic outcome measure to evaluate the quality of life, comprising 36 items distributed into eight subdimensions, which are physical functioning, general health, pain, mental health, social functioning, vitality, role limitations due to physical problems, and role limitations due to emotional problems. The scores of the subdimensions can be in the range from 0 to 100 , with lower scores indicating low quality of the life.

\section{Statistical Analysis}

SPSS for Windows version 17.0 software was used for the statistical analyses. We conducted descriptive analyses to summarize the patients' baseline characteristics (e.g., age, gender, body mass index and HGS) and presented the results in mean \pm standard deviation. Quantitative variables were tested for normality using the Shapiro Wilk test. The independent $t$-test was used for analyzing the normally distributed variables. The MannWhitney $\mathrm{U}$ test was used for testing the differences in 
TABLE 1. Basic characteristics of study subjects

\begin{tabular}{lccc} 
& $\begin{array}{c}\text { Sarkopenia with osteopenia } \\
(\mathrm{n}=37)\end{array}$ & $\begin{array}{c}\text { Sarkopenia with osteoporosis } \\
(\mathrm{n}=40)\end{array}$ & $\mathrm{p}$ \\
\hline Age $(\mathrm{year})$ & $71 \pm 4.5$ & $71.5 \pm 5.7$ & 0.94 \\
Gender $(\mathrm{F} / \mathrm{M})$ & $26 / 11$ & $33 / 7$ & 0.28 \\
BMI $\left(\mathrm{kg} / \mathrm{m}^{2}\right)$ & $30.4 \pm 7.4$ & $28.2 \pm 3.5$ & 0.13 \\
Serum vitamin D levels $(\mathrm{ng} / \mathrm{ml})$ & $20 \pm 11.2$ & $23.2 \pm 19.1$ & 0.92 \\
Hand grip strength $(\mathrm{kg})$ & $18.5 \pm 4.3$ & $15.8 \pm 3.8$ & $\mathbf{0 . 0 0 5}$ \\
Usual gait speed $(\mathrm{m} / \mathrm{s})$ & $0.55 \pm 0.2$ & $0.59 \pm 0.1$ & 0.70 \\
Skeletal muscle mass index $\left(\mathrm{kg} / \mathrm{m}^{2}\right)$ & $6.9 \pm 0.4$ & $6.78 \pm 0.1$ & 0.68 \\
\hline
\end{tabular}

BMI: Body mass index; *: Statistically significant at $p<0.05$

TABLE2. Results of balance testing

Biodex stability system

Sarkopenia with osteopenia

$(n=37)$

Sarkopenia with osteoporosis

$p$

\begin{tabular}{lccc}
\multicolumn{5}{c}{$(n=37)$} & $(n=40)$ & \\
\hline OSI & $4.03 \pm 1.71$ & $5.04 \pm 1.80$ & $\mathbf{0 . 0 1}^{*}$ \\
APSI & $3.27 \pm 1.29$ & $4.30 \pm 1.55$ & $\mathbf{0 . 0 0 2}^{*}$ \\
MLSI & $3.01 \pm 1.21$ & $3.71 \pm 1.71$ & $\mathbf{0 . 0 4}^{*}$ \\
\hline
\end{tabular}

APSI: Antero-Posterior Stability Index; MLSI: Medio-Lateral Stability Index; OSI: Overall Stability Index; *: Statistically significant at $p<0.05$.

balance indices (OSI, APSI, and MLSI). Gender differences between osteoporotic and osteopenic patients were tested with the chi-square test.

\section{RESULTS}

In this study, 77 patients with sarcopenia were included. The mean age of the participants was $71.3 \pm 5.1$ years ( $\mathrm{min}$ : 65 max: 85). The mean vitamin $\mathrm{D}$ levels of the patients were $21.7 \pm 15.8$ ( $\min : 3$ max: 73$) \mathrm{ng} / \mathrm{ml}$. The demographic data of the study patients are presented in Table 1.

The measures of balance in all tested directions were statistically significantly lower in the osteoporosis group $(\mathrm{p}=0.01, \mathrm{p}=0.002, \mathrm{p}=0.04$ for OSI, APSI, and MLSI, respectively) compared to the osteopenia group (Table 2).

The quality of life scores was statistically significantly lower in all subdimensions, excluding the mental health, in patients with combined osteoporosis and sarcopenia $(\mathrm{p}<0.05)$ compared to the patients with osteopenia and sarcopenia (Table 3).

\section{DISCUSSION}

The results of our study showed that the joint occurrence of osteoporosis and sarcopenia was related to a potential loss of balance and reduction in quality of life in the older population. The combination of osteopenia/osteoporosis and sarcopenia is more prevalent in the old age, predisposing the individuals to a high risk of falls, fractures, and eventual functional decline. To our knowledge, our study is the first study in quantifying the balance parameters objectively along with the quality of life in individuals with combined sarcopenia and osteopenia/osteoporosis. It is reported in the literature that loss of balance and a decline in the quality of life develop in old individuals when sarcopenia is accompanied by osteoporosis.

The pathophysiology of muscle and bone loss reveals overlapping features. There is an intense and complex interaction between these two tissue types mechanically (the mechanostat hypothesis) [15] and biochemically (e.g., with the involvement of estrogen, testosterone, GH, IGF-1, IL-6, and osteocalcin) [16]. Previous studies cat- 
TABLE 3. Health-related quality of life

\begin{tabular}{l} 
SF-36 subscales \\
\hline Physical functioning \\
Role limitations due to physical problems \\
Role limitations due to emotional problems \\
Vitality \\
Emotional well-being \\
Social functioning \\
Pain \\
General health
\end{tabular}

Sarkopenia with osteopenia $(n=37)$

$66.08 \pm 8.17$

$43.24 \pm 20.11$

$75.65 \pm 31.09$

$46.48 \pm 9.49$

$52.54 \pm 12.26$

$59.45 \pm 15.97$

$39.72 \pm 10.00$

$53.67 \pm 8.63$
Sarkopenia with osteoporosis $(n=40)$

$44.75 \pm 6.78$

$14.37 \pm 17.80$

$29.98 \pm 31.82$

$36.62 \pm 11.05$

$54.80 \pm 9.72$

$50.00 \pm 12.00$

$31.55 \pm 9.17$

$40.25 \pm 7.24$ $\mathrm{p}$

$0.00 *$

$0.00 *$

$0.00 *$

$0.00 *$

0.672

$0.00 *$

$0.00 *$

$0.00 *$

SF-36: Short-form-36; *: Statistically significant at $p<0.05$.

egorized individuals with combined osteopenia/osteoporosis and sarcopenia as osteosarcopenic (OS) or sarco-osteopenic due to the interactions between the bone and muscle tissue [17]. Yu et al. [18] have recently conducted a study on male osteosarcopenia patients, reporting a 3.5fold increased risk of fractures and they have reported that the risk is significantly higher compared to the presence of sarcopenia or osteopenia alone. Although this study was conducted only on males, it provided insight into potential additional risks associated with osteosarcopenia.

Drey et al. [19] reported that osteosarcopenia occurred much more prevalently compared to the presence of either sarcopenia and osteopenia alone, stressing that both the bone and muscle tissue should be treated in old individuals suffering from actual osteoporosis and fractures.

Balance is the postural fit performed either at rest or during activity to sustain the center of gravity on the supporting surface. The musculoskeletal system is a major contributor to maintain balance. Increasing age is associated with a reduction in the number of muscle fibers and a reduction in the number of cross-sections of the remaining fibers (fiber atrophy), leading to muscle weakness, which is a major factor involved in the loss of balance. Maintaining the balance during mobility in patients with sarcopenia becomes difficult due to the emerging coordination deficits resulting from the poor physical performance of the striated muscles executing this function. Although it has not been clarified yet whether the declining muscle strength is the cause or the result of osteoporosis, the relationship between muscle strength and bone mineral density (BMD) has been shown in studies [20]. In particular, the trunk muscle strength is important in maintaining the balance during the daily activities of life. A gradual immobilization developing in association with increasing age occurs due to a sedentary lifestyle and confers a risk factor for reductions in muscle strength and BMD [21].

Handgrip strength alone or in combination with BMD should be used for identifying the individuals having an increased risk of fractures [22]. Our study may conclude the same results, too, as HGS was significantly lower and the balance parameters were significantly poor in the patients with osteoporosis. Loss of balance can be considered as an increased risk factor for fractures.

The potential for having a low quality of life (QOL) is not surprising in old individuals diagnosed with sarcopenia. Several studies are available in the literature, evaluating the quality of life in individuals suffering from sarcopenia and osteoporosis. Although the disease-specific QOL scales are recommended for each of these disorders, we preferred to use a generic measure of QOL [23]. In the patients with osteoporosis, we found lower scores in all subdimensions of SF-36, excluding the mental health subcategory. There was not a significant difference between the osteopenic and osteoporotic groups in terms of the mental health scores and the scores were lower in both groups, compared to Turkish norms. This may suggest that not only physical health but also mental health is affected in the patients suffering from tissue loss both in bones and muscles.

There are several reasons to consider the potential beneficial effects of vitamin D on muscle and bone tissues, according to the results of the recent reviews performed by various experts and scientific societies [24, 25]. In our 
study, insufficient vitamin D levels were found in both groups; therefore, the observed differences in the balance parameters in both groups could not be associated with the lower vitamin $\mathrm{D}$ levels.

We recognize that our study has some limitations. The major limitation is the relatively small sample size. Secondly, we did not include any patients with sarcopenia alone; therefore, we could not perform any comparisons with individuals having a normal BMD.

In conclusion, we found balance loss in the old individuals with combined sarcopenia and a significant loss in bone mass, impairing their QOL in both physical and social aspects. Therefore, an assessment of bone parameters will identify individuals with a higher risk of fractures. Also, an assessment of muscle parameters will allow for screening for other major risk factors of general health. Further prospective long-term studies are warranted to confirm our study results and to develop assessment tools to evaluate sarcopenia along with the existing tools used for evaluating the fracture risk (such as FRAX Assessment Tool).

Ethics Committee Approval: The Inonu University Clinical Research Ethics Committee granted approval for this study (217/75).

Conflict of Interest: No conflict of interest was declared by the authors.

Financial Disclosure: The authors declared that this study has received no financial support.

Authorship Contributions: Concept - RB; Design - RB; Supervision - $\mathrm{RB}$; Materials - $\mathrm{RB}$, $\mathrm{BE}$; Data collection and/or processing $-\mathrm{RB}$, $\mathrm{SA}$; Analysis and/or interpretation $-\mathrm{RB}$, $\mathrm{SA}$; Literature review $-\mathrm{RB}$, $\mathrm{BE}$; Writing - RB; Critical review - YE.

\section{REFERENCES}

1. Burton LA, Sumukadas D. Optimal management of sarcopenia. Clin Interv Aging 2010;5:217-28. [CrossRef]

2. Cruz-Jentoft AJ, Landi F, Schneider SM, Zúñiga C, Arai H, Boirie Y, et al. Prevalence of and interventions for sarcopenia in ageing adults: a systematic review. Report of the International Sarcopenia Initiative (EWGSOP and IWGS). Age Ageing 2014;43:748-59. [CrossRef]

3. Yazar T, Yazar HO. Prevalance of sarcopenia according to decade. Clinical Nutrition ESPEN 2019;29:137-41. [CrossRef]

4. Bohannon RW, Williams Andrews A. Normal walking speed: a descriptive meta-analysis. Physiotherapy 2011;97:182-9. [CrossRef]

5. Cruz-Jentoft AJ, Baeyens JP, Bauer JM, Boirie Y, Cederholm T, Landi F, et al; European Working Group on Sarcopenia in Older People. Sarcopenia: European consensus on definition and diagnosis: Report of the European Working Group on Sarcopenia in Older People. Age Ageing 2010;39:412-23. [CrossRef]

6. Topinková E. Aging, disability and frailty. Ann Nutr Metab 2008;52 Suppl 1:6-11. [CrossRef]
7. Thornell LE, Lindström M, Renault V, Mouly V, Butler-Browne GS. Satellite cells and training in the elderly. Scand J Med Sci Sports 2003;13:48-55. [CrossRef]

8. Güngen C, Ertan T, Eker E, Yaşar R, Engin F. Reliability and validity of the standardized Mini Mental State Examination in the diagnosis of mild dementia in Turkish population. Turk Psikiyatri Derg 2002;13:273-81.

9. Aydog E, Depedibi R, Bal A, Eksioglu E, Unlü E, Cakci A. Dynamic postural balance in ankylosing spondylitis patients. Rheumatology (Oxford) 2006;45:445-8. [CrossRef]

10. World Health Organization. Assessment of fracture risk and its applications to screening for postmenopasual osteoporosis. WHO Technical Report Series 843. Genewa, Switzerland. 1994.

11. Massy-Westropp NM, Gill TK, Taylor AW, Bohannon RW, Hill CL. Hand Grip Strength: age and gender stratified normative data in a population-based study. BMC Res Notes 2011;4:127. [CrossRef]

12. Bahat G, Tufan A, Tufan F, Kilic C, Akpinar TS, Kose M, et al. Cutoff points to identify sarcopenia according to European Working Group on Sarcopenia in Older People (EWGSOP) definition. Clin Nutr 2016;35:1557-63. [CrossRef]

13. Iannuzzi-Sucich M, Prestwood KM, Kenny AM. Prevalence of sarcopenia and predictors of skeletal muscle mass in healthy, older men and women. J Gerontol A Biol Sci Med Sci 2002;57:M772-7. [CrossRef]

14. Demiral Y, Ergor G, Unal B, Semin S, Akvardar Y, Kivircik B, et al. Normative data and discriminative properties of short form 36 (SF-36) in Turkish urban population. BMC Public Health 2006;6:247. [CrossRef]

15. Isaacson J, Brotto M. Physiology of Mechanotransduction: How Do Muscle and Bone "Talk" to One Another? Clin Rev Bone Miner Metab 2014;12:77-85. [CrossRef]

16. Kawao N, Kaji H. Interactions between muscle tissues and bone metabolism. J Cell Biochem 2015;116:687-95. [CrossRef]

17. Kull M, Kallikorm R, Lember M. Impact of a new sarco-osteopenia definition on health-related quality of life in a population-based cohort in Northern Europe. J Clin Densitom 2012;15:32-8. [CrossRef]

18. Yu R, Leung J, Woo J. Incremental predictive value of sarcopenia for incident fracture in an elderly Chinese cohort: results from the Osteoporotic Fractures in Men (MrOs) Study. J Am Med Dir Assoc 2014;15:551-8.

19. Drey M, Sieber CC, Bertsch T, Bauer JM, Schmidmaier R; FiAT intervention group. Osteosarcopenia is more than sarcopenia and osteopenia alone. Aging Clin Exp Res 2016;28:895-9. [CrossRef]

20. Iki M, Saito Y, Kajita E, Nishino H, Kusaka Y. Trunk muscle strength is a strong predictor of bone loss in postmenopausal women. Clin Orthop Relat Res 2006;443:66-72. [CrossRef]

21. Sinaki M, Brey RH, Hughes CA, Larson DR, Kaufman KR. Balance disorder and increased risk of falls in osteoporosis and kyphosis: significance of kyphotic posture and muscle strength. Osteoporos Int 2005;16:100410. [CrossRef]

22. Kärkkäinen M, Rikkonen T, Kröger H, Sirola J, Tuppurainen M, Salovaara $\mathrm{K}$, et al. Physical tests for patient selection for bone mineral density measurements in postmenopausal women. Bone 2009;44:660-5.

23. Tsekoura M, Kastrinis A, Katsoulaki M, Billis E, Gliatis J. Sarcopenia and Its Impact on Quality of Life. Adv Exp Med Biol 2017;987:213-8.

24. Rizzoli R, Stevenson JC, Bauer JM, van Loon LJ, Walrand S, Kanis JA, et al; ESCEO Task Force. The role of dietary protein and vitamin $\mathrm{D}$ in maintaining musculoskeletal health in postmenopausal women: a consensus statement from the European Society for Clinical and Economic Aspects of Osteoporosis and Osteoarthritis (ESCEO). Maturitas 2014;79:122-32. [CrossRef]

25. Halfon M, Phan O, Teta D. Vitamin D: a review on its effects on muscle strength, the risk of fall, and frailty. Biomed Res Int 2015;2015:953241. 